\title{
Vicarious responses to pain in anterior cingulate cortex: Is empathy a multisensory issue?
}

\author{
INDIA MORRISON \\ University of Wales, Bangor, Wales \\ DONNA LLOYD \\ University of Liverpool, Liverpool, England \\ GIUSEPPE DI PELLEGRINO \\ University of Urbino, Urbino, Italy \\ and \\ NEIL ROBERTS \\ University of Liverpool, Liverpool, England
}

\begin{abstract}
Results obtained with functional magnetic resonance imaging show that both feeling a moderately painful pinprick stimulus to the fingertips and witnessing another person's hand undergo similar stimulation are associated with common activity in a pain-related area in the right dorsal anterior cingulate cortex (ACC). Common activity in response to noxious tactile and visual stimulation was restricted to the right inferior Brodmann's area 24b. These results suggest a shared neural substrate for felt and seen pain for aversive ecological events happening to strangers and in the absence of overt symbolic cues. In contrast to ACC $24 \mathrm{~b}$, the primary somatosensory cortex showed significant activations in response to both noxious and innocuous tactile, but not visual, stimuli. The different response patterns in the two areas are consistent with the ACC's role in coding the motivational-affective dimension of pain, which is associated with the preparation of behavioral responses to aversive events.
\end{abstract}

When once compassion is stirred within me by another's pain, then his weal and woe go straight to my heart, exactly in the same way, if not always to the same degree, as otherwise I feel only my own. Consequently the difference between myself and him is no longer an absolute one.

-Arthur Schopenhauer,

On the Basis of Morality (1841)

The sensation one gets upon watching another person get hurt has probably happened to all of us: It is something that makes us recoil, cringe, wince, say "ouch!" or experience feelings otherwise associated with pain, even if we are sitting snugly in an armchair at a safe remove from any harm. Although familiar to most people, this variety of experience is not easily described in empirical terms and is usually called empathy or sympathy in everyday language. Here we refer to the sensations that arise with regard to the perceived physical pain of others as vicarious pain. Vicarious pain may be a crucial predicate for more sophisticated forms of empathy, such as helping and offering comfort, and perhaps even for such complex

The authors thank Paul Downing, John Parkinson, Francis McGlone, Justin Williams, and two anonymous reviewers for valuable comments. We also thank Phillipa Walker, Sarah Wilson, Arshad Zaman, and the radiographers at the Walton Centre for Neurology, Liverpool.Correspondence concerning this article should be addressed to I. Morrison, Centre for Cognitive Neuroscience, School of Psychology, University of Wales, Bangor, Gwynedd LL57 2AS, U.K. (e-mail: pspc46@bangor.ac.uk). cognitive processes as moral reasoning. When one reacts to another person's predicament as if one were in that position oneself, processes are taking place in the brain that may facilitate an immediate grasp of that person's emotional state.

However, thinkers pondering the nature of empathy have noticed a tangled problem at its core, which has been referred to as the paradox of sympathy (Wispé, 1991). The paradox is this: If all that is available to us is third-person information about someone else's situation or emotional state, how can that produce what we readily identify as a similar first-person subjective state within ourselves? Put in other terms, this paradox can be broken down into two related questions. The first is a question of mechanism; that is, how does the brain accomplish this? The second is a motivational question, having to do with the behavioral relevance to an observer of another person's distress: We may understand what is happening, but what makes us care about it?

Recently, neuroscience has begun to reveal mechanisms that could throw light upon the first of these questions and thus provide insight into the second. Several neuroimaging studies have supported the view that an immediate, subjective interpretation of another person's particular emotional state is accompanied by the activation of regions directly involved in the production of that emotion (Carr, Iacoboni, Dubeau, Mazziotta, \& Lenzi, 
2003; Decety \& Chaminade, 2003; Phillips et al., 1997; Wicker et al., 2003). This perspective is bolstered by a growing body of research indicating that the observation of others' actions engages circuits involved in the preparation and planning of self-generated motor actions (di Pellegrino, Fadiga, Fogassi, Gallese, \& Rizzolatti, 1992; Hari et al., 1998; Iacoboni et al., 1999; Rizzolatti, Fadiga, Fogassi, \& Gallese, 1999; Rizzolatti, Fadiga, Gallese, \& Fogassi, 1996). The existence of such actionperception mechanisms has provided the foundation for a recent model of empathy which integrates emotional, behavioral, and cognitive aspects of interpersonal phenomena (Preston \& de Waal, 2002).

A similar unifying basis has so far been little investigated with respect to the mechanisms of pain processing. A notable exception is a recent functional magnetic resonance imaging (fMRI) study showing that affective pain-related areas such as the dorsal anterior cingulate cortex (ACC) and anterior insula can be activated by a visual signal indicating that a loved one will receive a painful electric shock (Singer et al., 2004). In this study, each female participant viewed her own hand alongside that of her established romantic partner as electrode shocks were delivered to one or the other at either low or high levels of stimulation. Visual cues projected onto a screen indicated to the participant whether the shock would occur to herself or to her partner, as well as whether the stimulation would be low (not painful) or high (painful). This study demonstrated that affect-related regions of a pain network can be engaged in situations in which there is an imminent and ongoing threat of pain both to oneself and to a loved one.

More specific indications come from earlier single-cell data of pain-related processing in human neurological patients (Hutchison, Davis, Lozano, Tasker, \& Dostrovsky, 1999). This study investigated pain-related responses in the ACC in 11 individuals undergoing cingulotomy surgery for the treatment of obsessive-compulsive disorder or severe depression. Using microelectrodes, Hutchison et al. recorded from the ACC as several types of painful stimuli were applied to the patients' hands (painful heat, painful cold, and mechanical pinpricks from a sharp probe). They found stimulus-specific pain responses in area $24 b$ of the dorsal ACC (24b' of Vogt, Nimchinsky, Vogt, \& Hof, 1995), including cells that discharged preferentially to the pinprick stimulus. One of these cells responded to the pinprick whether it was administered to the patient's own hand or to that of the experimenter. This particular cell appears to have been sensitive not only to painrelated input originating from the hand, but also to visual input carrying information about another person's hand.

Evidence surrounding vicarious pain mechanisms from neurological case studies is quite scant, but one unusual case that may have bearing on the pathological representation of others' pain was reported anecdotally in a letter (Bradshaw \& Mattingley, 2001). A deceased patient's widow described to the authors an unusual symptom of her husband's allodynia (a condition in which non-noxious touch is painful). When she herself would experience a sudden minor injury such as knocking her hand against a table, he would become very agitated, claiming that it hurt him to witness such accidents. Unfortunately, no CT scans exist of the extent of the damage in the man's brain or the areas affected. It is possible only to speculate about what might have caused his symptoms, but perhaps the damage altering the representation of his own sensations had a corresponding impact upon his representation of others' sensations as well. However, a combined positron emission tomography (PET) and fMRI investigation of another allodynia patient, in whom symptoms persisted despite a bifocal infarct in both the primary somatosensory cortex (SI) and the right ACC, suggests that any possible cortical substrates of allodynia are complex and not isolable to a single circumscribed region in the ACC or elsewhere (Peyron, Garcia-Larrea, et al., 2000).

Taken together, the available neuroimaging and neurophysiological evidence raises the possibility that merely observing another person in a painful situation can give rise to a pain-related response in the ACC. In the present study, we used fMRI to test the hypothesis that painful stimulation increases bloodflow in ACC $24 \mathrm{~b}$ of normal individuals, not only during the firsthand experience of an ecologically relevant mechanical stimulus (pinprick), but also during the observation of another individual undergoing similar stimulation. Such a common neural substrate for felt and vicarious pain would address the question of mechanism posed by the "paradox of sympathy" mentioned above.

Other studies have shown a dissociation between the sensory-discriminative and motivational-affective dimensions of pain processing. In the sensory-discriminative dimension, the SI encodes sensory components of a painful stimulus, such as the bodily location and intensity of the stimulus; in the motivational-affective dimension, the ACC contributes to evaluation, subjective discomfort, and response preparation in the context of painful or aversive stimuli (Craig, 2003; Devinsky, Morrell, \& Vogt, 1995; Melzack, 1999; Rainville, Carrier, Hofbauer, Bushnell, \& Duncan, 1999; Sewards \& Sewards, 2002). To determine whether a similar dissociation held in our own study, blood flow responses to noxious and innocuous tactile and visual stimuli were compared in right ACC $24 \mathrm{~b}$, and in a region of interest (ROI) on the postcentral gyrus corresponding to SI area $3 \mathrm{~b} / 1$ contralateral to the stimulated hand. Differences in response patterns to sensory aspects (e.g., tactile) and motivational aspects (e.g., noxiousness) between the SI and the ACC would reinforce the distinctive roles for these areas in sensory-discriminative and motivational-affective dimensions of pain processing, respectively.

\section{METHOD}

\section{Participants and Experimental Design}

Functional MRI (1.5 T; 24 slices; $5 \mathrm{~mm}$ thickness; TR $=3 \mathrm{sec})$ was used to compare the responses of 14 healthy participants ( 9 female, 5 male; mean age 23 years; 9 right-handed, 5 left-handed) as they experienced unpleasant pricks to the fingertips and as they 
viewed video clips of others being similarly pricked. Data were also collected for control conditions involving innocuous touch presented in both the tactile and visual modalities. The stimulus for the experienced pain condition was a mildly painful prick to the middle finger of the left hand using a nonferromagnetic sharp probe $(\sim 1 \mathrm{~Hz} / 15 \mathrm{sec})$. During scanning, the hand was placed palm-up in a relaxed position, out of the participant's sight. The tactile control stimulus was a cotton bud (Q-tip) similarly pressed onto the fingertip.

For the observed pain condition, a video featuring a model's left hand being pricked on the finger with a hypodermic needle was displayed. The video featured the needle coming into contact with the hand and excluded the model's face. The visual control video was identical except for the substitution of a cotton bud for the needle. Placement of the sharp probe in a plasticine-filled syringe increased the visual resemblance between it and the hypodermic needle in the video. The participants were familiarized with the sharp probe prior to scanning, but during scanning they could neither see their hands nor the stimulus being applied. The videos were projected onto a screen at the participants' feet as they looked into a mirror.

All visual stimuli were presented on a laptop computer using Presentation software (Version 0.70, www.neurobs.com). The observed pain and visual control stimuli were presented in a trial identical in design to the tactile run. The observed and experienced pain experimental runs were conducted separately. Each run consisted of five blocks of 15 -sec presentations of both the painful and neutral stimuli interspersed with $15 \mathrm{sec}$ of baseline rest, giving a total scan time of approximately $5 \mathrm{~min}$. For every condition, there was a total of five stimulus presentations. After scanning, participants were asked to rate the unpleasantness of both the experienced and the observed stimuli, respectively, on a scale of 1 to 5, ranging from not at all unpleasant to extremely unpleasant.

\section{Analysis}

Analysis was carried out using FEAT (fMRI Expert Analysis Tool) Version 5.00, part of the FMRIB software library (FSL-Oxford Centre for Functional Magnetic Resonance Imaging of the Brain; www.fmrib.ox.ac.uk/fsl). The following prestatistics processing was applied: motion correction using MCFLIRT (Jenkinson, Bannister, Brady, \& Smith, 2002); nonbrain removal using BET (Smith, 2002); spatial smoothing using a Gaussian kernel of FWHM $5 \mathrm{~mm}$; mean-based intensity normalization of all volumes by the same factor; and highpass temporal filtering (Gaussian-weighted LSF straightline fitting, with $\sigma=30.0 \mathrm{sec}$ ). Time-series statistical analysis was carried out using FILM (FMRIB's Improved Linear Model) with local autocorrelation correction (Woolrich, Ripley, Brady, \& Smith, 2001). Z (Gaussianized T/F) statistic images were thresholded using clusters determined by $Z>1.8$ and a (corrected) cluster significance threshold of $p=.05$ (Forman et al., 1995; Friston, Worsley, Frackowiak, Mazziotta, \& Evans, 1994; Worsley, Evans, Marrett, \& Neelin, 1992). Registration to high-resolution and/or standard images was carried out using FLIRT (FMRIB's Linear Image Registration Tool; Jenkinson \& Smith, 2001; Jenkinson et al., 2002).

\section{RESULTS}

\section{Whole Brain Contrasts}

Feeling the sharp probe elicited significant activations in cortical areas consistently implicated in imaging investigations of pain (Table 1; Peyron, Laurent, \& GarciaLarrea, 2000). Peak clusters in group-averaged data fell in the left insula, the contralateral primary and secondary (SII) somatosensory cortices, and the left (ipsilateral) cerebellum. Significant peaks in these areas and the right (contralateral) medial thalamus remained after subtraction of the tactile control eliminated the signal resulting
Table 1

Foci of Pain-Related Activation

During Experience and Observation

\begin{tabular}{ccc}
\hline & $\begin{array}{c}\text { Coordinates of } \\
\text { Peak Activation } \\
(x, y, z, \text { in mm })\end{array}$ & Max \\
Brain Regions & & \\
\hline
\end{tabular}

Main Effect of Experienced Pain (Pain-Rest)

Right inferior parietal lobule

Left insula

Right parietal postcentral gyrus

Left cerebellum

$\begin{array}{ll}70,-24,26 & 5.18 \\ -46,-6,0 & 5.05 \\ 62,-16,38 & 4.80 \\ -18,-56,-30 & 5.18\end{array}$

Pain Compared With Neutral Stimulus (Pain-Neutral)

Right parietal postcentral gyrus

ACC/pre-SMA

Left parietal postcentral gyrus

Right frontal precentral gyrus

Right medial thalamus

$62,-16,22$

$0,-8,58$

5.13

$-58,-24,14$

5.12

$32,-20,58$

$16,-14,2$

4.77

4.81

4.80

Main Effect of Observed Pain (Pain-Rest)

Right ACC

$2,42,16$

Right medial frontal gyrus

Left ACC

Left superior frontal gyrus

$6,52,2$

$-8,-2,32$

4.72

4.40

4.09

3.94

Conjunction Analysis

[(Pain Experience-Rest) + (Pain Observation-Rest)]

Right ACC

$6,0,32$

4.40

Note-All values $p<.05$, corrected.

from stimulation of non-nociceptive tactile receptors. The main effect of pain observation revealed activity in the anterofrontal and medial frontal regions including the cingulate gyrus, whereas the neutral visual stimulus failed to produce activations above the threshold level. A conjunction analysis showing common areas of significant activation between the main effects of pain experience and observation compared to a resting baseline revealed a significant cluster in the right inferior ACC area $24 \mathrm{~b}(x=6 \mathrm{~mm}, y=0 \mathrm{~mm}, z=32 \mathrm{~mm})$ common to the two conditions, reflecting shared activity correlated with both feeling and seeing the noxious stimulus (Figure 1).

\section{ROI Analyses}

Anterior cingulate area 24b. The anatomical definition of the ROI was based on Talairach coordinates reported in Hutchison et al.'s (1999) previous single-unit study, which also encompasses the site of overlap between the experience and observation conditions in our study $(x=3-5 \mathrm{~mm}, y=3-13 \mathrm{~mm}, z=26-36 \mathrm{~mm})$ (Figure 1A). This region corresponds to the right dorsal Brodmann's area (BA) 24, area $24 b^{\prime}$ of Vogt et al. (1995). Within this region, the average percent signal change was significantly greater $(p<.001)$ for the pain conditions than for the control conditions, irrespective of whether the stimulus was felt or seen (Figure 1B).

Primary somatosensory $3 \mathbf{b} / \mathbf{1}$. An ROI in the right (contralateral) SI was defined by the coordinates of the most significant cluster in the main effect for the innocuous tactile stimulus ( $x=64, y=-16, z=28$ ) (not shown in Table 1). This activation fell on a region of postcentral gyrus most likely corresponding to the hand 
A
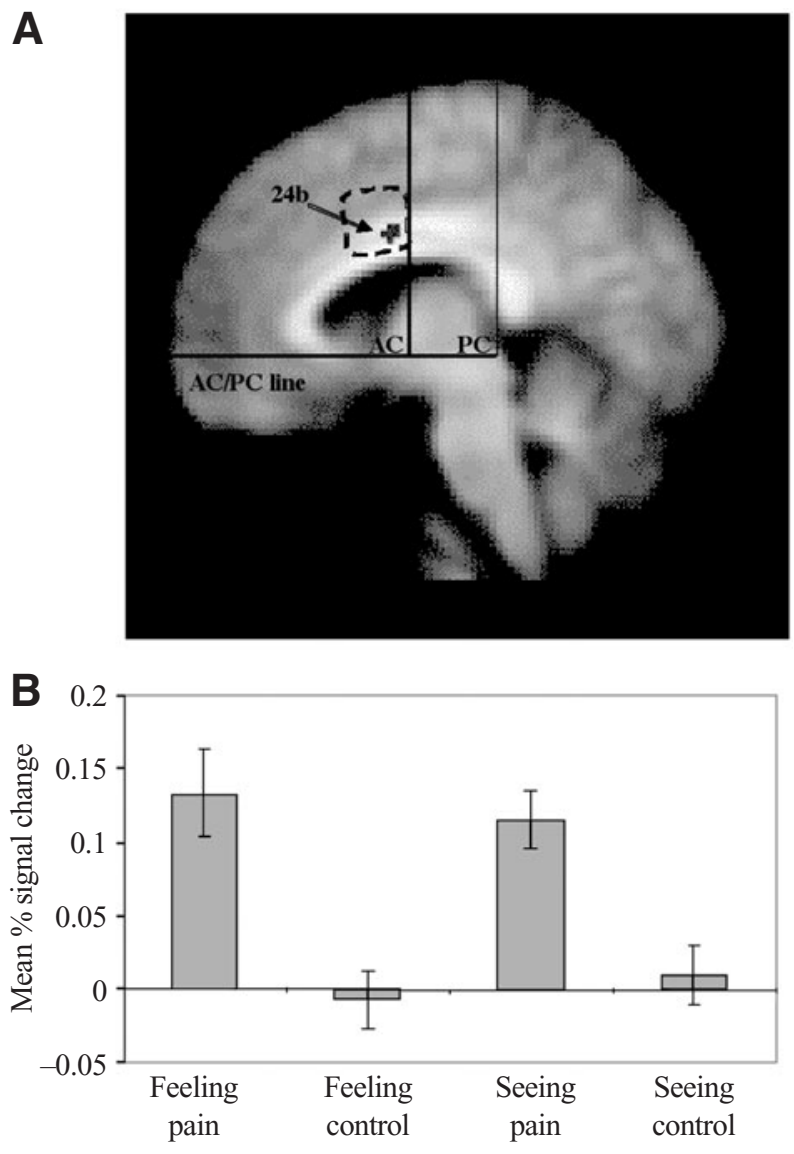

Figure 1. Activation in the $\mathrm{ACC}$ in response to sharp probe stimulation delivered in the tactile and visual modalities. (A) Sagittal slice showing the common activation between the main effects of feeling the sharp probe versus seeing it applied to someone else $(x, y, z=6,0,32)$. Group functional data are superimposed upon a T1-weighted normalized anatomical image for 14 participants. Dashed line indicates the region of interest defined by the singleunit recording site reported in Hutchison et al. (1999). AC $=$ anterior commissure, $\mathrm{PC}=$ posterior commissure. (B) Signal modulation in a region of interest in $A C C$ 24b defined by the coordinate range reported in Hutchison et al. $(x=3-5 \mathrm{~mm}, y=$ 2-4 mm, $z=32 \mathrm{~mm}$ ). Mean percent signal change was significantly greater in the pain conditions than in the control conditions (see Results).

area $3 \mathrm{~b} / 1$. This showed significant activations to both noxious and innocuous tactile stimuli but not to visual stimuli $(p<.001)$ (Figure 2). The difference between the tactile activations in the SI was not significant $(p=$ $.60)$.

Comparison of ACC and SI ROIs. The SI ROI showed a significantly greater response to the innocuous tactile stimulus than did the ACC ROI $(p<.001)$. In contrast, the noxious visual stimulus elicited a greater response in the ACC than in the SI $(p<.001)$. Mean percent signal changes for the innocuous visual stimulus were at or below baseline for both the ACC and the SI (Figure 2). The response to the sharp probe in the SI was significantly greater than the tactile pain-related re- sponse in the ACC $(p<.001)$, although both activations were significant in the higher level group analysis ( $p=$ .05 , corrected).

\section{Unpleasantness Ratings}

Ratings were collected from 13 of the 14 participants after scanning. The participants consistently rated the observed unpleasantness (how unpleasant did it look?) higher than the experienced unpleasantness (how unpleasant did it feel?) of the visual and tactile conditions, respectively. On a scale of $1-5$, ranging from not at all unpleasant to extremely unpleasant, the mean score for feeling the sharp probe was 2.15; for seeing the pinprick video, it was 3.15 . This difference was not significant $(p=.10)$.

\section{DISCUSSION}

These findings corroborate single-unit evidence (Hutchison et al., 1999) and point to a unique role for the right ACC $24 \mathrm{~b}$ in vicarious pain. Our results are also consistent with other studies demonstrating the participation of the dorsal ACC in either experienced and observed pain, or both (Jackson, Meltzoff, \& Decety, 2004; Singer et al., 2004), and with neuroimaging results implicating the ACC in the appraisal of one's own and others' distress (Eisenberger, Lieberman, \& Williams, 2003; Peyron, Laurent, \& Garcia-Larrea, 2000; Singer et al., 2004). Furthermore, a comparison of mean percent signal changes in the right ACC and SI ROIs showed significant differences between responses to the innocuous tactile stimuli and noxious visual stimuli. The ACC modulation corresponded to noxious aspects of the stimuli, regardless of whether they were presented in the tactile or visual modality, whereas the SI responses corresponded to tactile but not visual elements of the stimuli, regardless of noxiousness.

\section{A Common Neural Substrate for Felt and Seen Pain}

The main result of this study was a focal overlap of activity in a pain-related area of the ACC, the right area $24 \mathrm{~b}$ ( $24 b^{\prime}$ of Vogt et al., 1995), suggesting a common neural substrate for felt and seen pain. Such shared activity provides a potential mechanism for the rapid subjective appraisal, in pain-related terms, of tissue-damaging events happening to others. It may also serve as a springboard for further neuroscientific study of the phenomenon of vicarious pain, as well as of more sophisticated processes or outcomes of empathy which may rely on such a mechanism. These results affirm previous neurophysiological and neuroimaging observations that nociceptive processing in the ACC, and area 24 in particular, can utilize visual information in its encoding of pain (Hutchison et al., 1999; Jackson et al., 2004; Singer et al., 2004).

The participants' own hands were not visible to them, and they were instructed to close their eyes during the "felt pain" condition. This allowed a dissociation be- 

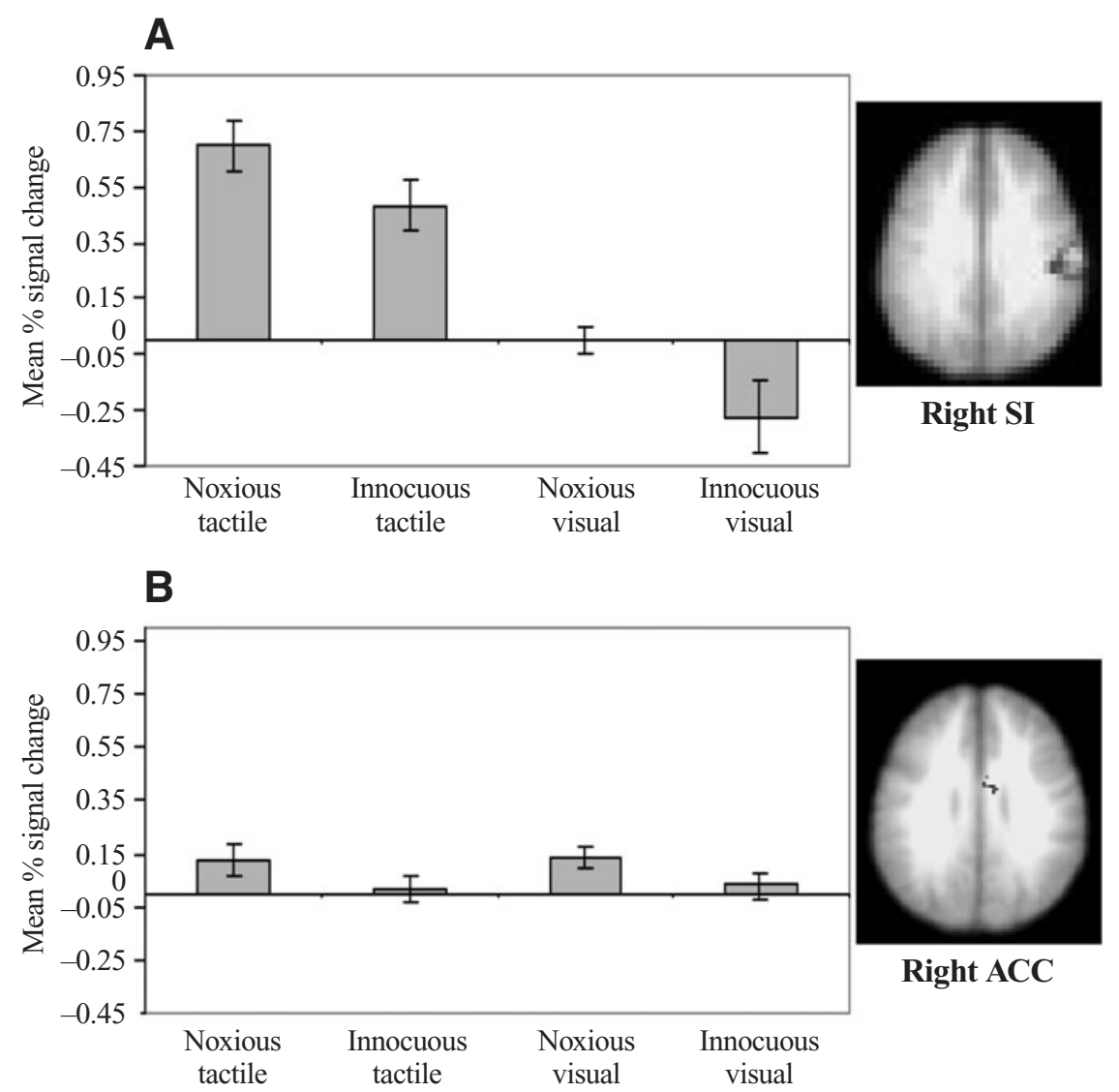

Figure 2. Differential responses in somatosensory and anterior cingulate cortices to noxious and innocuous tactile and visual stimuli. (A) Preferential modulation within cluster in right primary somatosensory hand area $(x, y, z=64,-16,28)$ to tactile noxious and innocuous stimuli. (B) Preferential modulation within cluster of right anterior cingulate cortex $(x, y, z=3,4,32)$ to noxious tactile and visual stimuli. Clusters are sample clusters representing activation within regions of interest. SI image: (innocuous tactile - rest); ACC image: (noxious tactile - rest) + (noxious visual - rest).

tween nociceptive/tactile and visual perception of the painful stimuli and an analysis of the differential contributions of each modality. This dissociation makes it possible to confirm that ACC $24 \mathrm{~b}$ is capable of integrating pain-related information independently of visual information about one's own hand in the firsthand experience of pain, rather than being a predominantly visually guided area.

The experiment differs from a recent neuroimaging study of empathy (Singer et al., 2004) in several crucial respects. Most notably, the participants were given no overt or arbitrary cue indicating the painful stimulation of the other person, but observed the needle coming directly into contact with the fingertip, distending the skin. Also, the models whose hands were featured in the videos were unknown to the participants, implying that vicarious pain effects do not depend on a longstanding relationship with the other person. A sharp, needlelike probe rather than an electrode was used as a painful stimulus. Although electrode stimulation more effectively elicits activation of nociceptive pathways, the needle stimulus was used here partly to recreate as well as possible the conditions of Hutchison et al.'s (1999) study and partly to maintain ecological validity in the stimulus videos.

It is conceivable that a function of visually cued responses in area $24 \mathrm{~b}$ is to apprehend potential threats, whether it is oneself or someone else who stands to be hurt. Areas of the ACC that represent pain affect are also active in anticipation of painful stimuli (Hsieh, StoneElander, \& Ingvar, 1999; Koyama, Tanaka, \& Mikami, 1998; Porro, Cettolo, Francescato, \& Baraldi, 2003), including stimulus-specific anticipatory discharge of neurons in area 24b (Hutchison et al., 1999). The relationship between anticipation and empathy in visually cued pain representations in the ACC may thus be a very close one, both functionally and subjectively. As such, it may even be fruitful to regard the representation of others' pain as a special case of anticipation.

Previous studies have shown the dorsal ACC to be implicated in attention and arousal (Downar, Crawley, 
Mikulis, \& Davis, 2002), especially when related to response preparation (Milham, Banich, Claus, \& Cohen, 2003). However, peak activations in studies of attention and emotional arousal tend to fall more anteriorly and superiorly than does the focus in this study, as, for example, in BA 32 or the more rostral portions of BA 24/25 (Keightley et al., 2003; Yamasaki, LaBar, \& McCarthy, 2002), which are larger in spatial extent and do not respond to painful stimulation (Davis, Taylor, Crawley, Wood, \& Mikulis, 1997). In our study, common activation in the ACC was restricted to $24 \mathrm{~b}$ and did not extend into these other areas.

\section{Vicarious Pain as a Motivational-Affective Representation}

We interpreted the profile of modulation in ACC $24 \mathrm{~b}$ as indicative of a motivational, rather than a sensory, role in vicarious pain. Various studies have implicated the ACC in motivation (Bush, Lu, \& Posner, 2000; Bush et al., 2002; Devinsky et al., 1995; Hadland, Rushworth, Gaffan, \& Passingham, 2003a), emotion and social behavior (Bush et al., 2000; Eisenberger et al., 2003; Hadland, Rushworth, Gaffan, \& Passingham, 2003b), and response selection (Hadland et al., 2003a; Paus, Petrides, Evans, \& Meyer, 1993; Rushworth, Hadland, \& Passingham, 2003; Walton, Bannerman, Alterescu, \& Rushworth, 2003). Motivational aspects of pain are those that pertain to desires, urges, or impulses to avoid or terminate a painful experience (Craig, 2003; Sewards \& Sewards, 2002).

Motivational-affective processing is associated primarily with nociceptive pathways ascending from the dorsal horn of the spinal cord through the medial thalamic nuclei, which send projections to the ACC (Craig, 2003; Devinsky et al., 1995; Peyron, Laurent, \& Garcia-Larrea, 2000; Vogt \& Sikes, 2000). In the ACC, nociceptive neurons are interspersed among cells that code for the aversive value of the stimulus (Koyama, Kato, Tanaka, \& Mikami, 2001; Porro et al., 2003). Projections from the anterior cingulate of area $24 \mathrm{~b}$ reach supplementary motor, premotor, cingulate motor, and primary motor cortices, influencing the selection of skeletomotor responses to painful stimuli (Devinsky et al., 1995; Matelli, Luppino, \& Rizzolatti, 1991; Vogt et al., 1995). Nociceptive fields in the ACC are thus taken to represent a motivational aspect of somatic pain, contributing to the mobilization and execution of volitional movements of aversion (Schnitzler $\&$ Ploner, 2000; Sewards \& Sewards, 2002). The results of the present study support this view, especially in light of the premotor activations alongside the $\mathrm{ACC}$ when pain experience was compared to the neutral tactile stimulus.

The motivational-affective dimension of pain processing is to a large extent functionally distinct from the sensory-discriminative dimension, which concerns somatotopic localization, intensity coding, discrimination of the type of painful sensation (e.g., burning, aching, stinging), and temporal characteristics such as its onset and offset (Hofbauer, Rainville, Duncan, \& Bushnell, 2001; Ploner, Freund, \& Schnitzler, 1999; Rainville, 2002; Rainville et al., 1999; Rainville, Duncan, Price, Carrier, \& Bushnell, 1997). The sensory-discriminative dimension is associated with nociceptive pathways ascending through the lateral thalamic nuclei and projecting to somatosensory cortices, including hand areas $3 \mathrm{~b}$ and 1 (Kenshalo, Iwata, Sholas, \& Thomas, 2000; Schnitzler, Seitz, \& Freund, 2000; Timmermann et al., 2001).

A relevant case study (Ploner et al., 1999) reports a patient with selective damage to the right postcentral gyrus and parietal operculum, the hand area of the SI and SII. When stimulated with a laser on the skin of the hands and feet, the patient was unable to localize a painful stimulus on the left hand but appeared to have intact motivational processing. He identified the painful sensation as "something he wanted to avoid," although he could not discriminate its sensory characteristics (Ploner et al., 1999). Conversely, stimulation of the anterior cingulate cortex in humans produces reports of unspecific motivations or urges, and feelings of "wanting or planning to do something" (Bancaud \& Talairach, 1992). Damage to the ACC, a cortical target for medial fiber projections, can alter pain perception without impairing localization, yet microstimulation does not produce feelings of pain (Davis, Hutchison, Lozano, \& Dostrovsky, 1994; Hutchison et al., 1999).

To investigate any similar dimensional dissociation in our data, we compared mean percent signal changes in right ACC 24b with those in a region of the postcentral gyrus corresponding to primary somatosensory hand area $3 \mathrm{~b} / 1$. Areas $3 \mathrm{~b}$ and 1 are directly adjacent (Gelnar, Krauss, Szeverenyi, \& Apkarian, 1998; Powell \& Mountcastle, 1959), and both are associated with the discrimination of passive tactile stimulation on the skin surface (Burton, MacLeod, Videen, \& Raichle, 1997; Kaas \& Collins, 2001; McGlone et al., 2002), as well as cutaneous representation of the digits of the contralateral hand (Blankenburg, Ruben, Meyer, Schwiemann, \& Villringer, 2003; Francis et al., 2000; Gelnar et al., 1998; Ringler, Greiner, Kohlloeffel, Handwerker, \& Forster, 2003). The SI ROI was defined on the basis of its significant response to the innocuous tactile stimulus under the assumption that activation here reflects a localized sensory response to stimulation of the contralateral hand.

The SI showed higher responses to both noxious and innocuous tactile stimuli, but not to visual stimuli, when compared to a resting baseline. The ACC showed a pattern of response that was higher to noxious stimuli regardless of sensory modality, but not to innocuous tactile or visual stimuli (Figure 2). These differences suggest that the vicarious pain effect observed in right ACC $24 \mathrm{~b}$ was more closely associated with the motivational than the sensory properties of the stimulus. They are also in accordance with other pain empathy studies in which a somatosensory contribution to vicarious pain was lacking (Jackson et al., 2004; Singer et al., 2004). 


\section{"Visuo-Nociceptive" Selectivity in the ACC: Analogy With Premotor Mirror Neurons}

This study demonstrates that the mere observation of a sharp object approaching a hand, making contact with it and distending the skin, is sufficient to engage a specific pain-related area in the ACC. The dorsal ACC receives indirect projections from superior temporal areas associated with higher level, semantic visual processing (Vogt \& Pandya, 1987), a region also important in associative and multisensory processing of information from different sensory modalities (Calvert, Campbell, \& Brammer, 2000; Calvert, Hansen, Iversen, \& Brammer, 2001; Hikosaka, 1997).

Pain-related areas in the ACC have extensive output connections to premotor and motor areas, as noted above. In this respect, ACC $24 \mathrm{~b}$ has several formal similarities to the properties of mirror neurons discovered in areas of macaque monkey premotor and parietal cortex (di Pellegrino et al., 1992; Rizzolatti et al., 1996), prompting an analogy between the functional organization of action recognition and that of the motivational-affective encoding of aversive third-person events.

Neural populations in macaque premotor F5 and parietal PF transform visual shape- and space-related object information into a motor-specific vocabulary of potential actions (Rizzolatti \& Luppino, 2001). These transformations are based on object features or other relevant cues, or, in the case of mirror neurons, upon the observation of others. Whereas in these fronto-parietal circuits, perception-action transformations are processed in kinesthetic-pragmatic terms, medial frontal circuits including the anterior cingulate area $24 \mathrm{~b}$ may code analogous transformations in terms of affective and motivational significance. Whether neurons in ACC $24 \mathrm{~b}$ can be considered "affective mirror neurons" remains to be seen, but the results of this study illustrate the strong possibility that a "mirror neuron principle" is not limited to kinesthetic action-perception circuits (Gallese, 2001, 2003), but may be at work in affective-motivational circuits as well.

\section{SUMMARY AND CONCLUSIONS}

A fundamental question about empathy concerns the neural correlates of our ability to understand the emotional states of others in immediate terms. To address this issue, fMRI was used to measure brain activation in normal participants while they either underwent moderately painful pinpricks to the fingertips or viewed a video of another person experiencing a similar stimulus. Both being pricked and observing another person being pricked was associated with focal activation of right inferior ACC $24 b$ (24b' of Vogt et al., 1995). Differences in the coding of noxious and tactile properties between the ACC and the SI support a dissociation between the motivationalaffective and sensory-discriminative dimensions of pain processing.
The organizational feature that enables the processing of visual information about painful events that befall others, even when they pose no immediate threat to the observer, admits an analogy with mirror neurons in the premotor cortex. Taken together, these results encroach on the age-old "paradox of sympathy" by providing a mechanism connecting observed painful events to an egocentric emotional and motivational network. Perhaps, although we cannot directly detect another person's tissue damage, we can still feel the suffering it causes.

\section{REFERENCES}

Bancaud, J., \& Talairach, J. (1992). Clinical semiology of frontal lobe seizures. Advances in Neurology, 57, 3-58.

Blankenburg, F., Ruben, J., Meyer, R., Schwiemann, J., \& VillRINGER, A. (2003). Evidence for a rostral-to-caudal somatotopic organization in human primary somatosensory cortex with mirrorreversal in areas $3 \mathrm{~b}$ and 1. Cerebral Cortex, 13, 987-993.

Bradshaw, J. L., \& Mattingley, J. B. (2001). Allodynia: A sensory analogue of motor mirror neurons in a hyperaesthetic patient reporting instantaneous discomfort to another's perceived sudden minor injury? Journal of Neurology, Neurosurgery, \& Psychiatry, 70, 135-136.

Burton, H., Macleod, A. M., Videen, T. O., \& Raichle, M. E. (1997). Multiple foci in parietal and frontal cortex activated by rubbing embossed grating patterns across fingerpads: A positron emission tomography study in humans. Cerebral Cortex, 7, 3-17.

Bush, G., LuU, P., \& Posner, M. I. (2000). Cognitive and emotional influences in anterior cingulate cortex. Trends in Cognitive Sciences, $\underline{\mathbf{4}}_{2}$ 215-222.

Bush, G., Vogt, B. A., Holmes, J., Dale, A. M., Greve, D., Jenike, M. A., \& Rosen, B. R. (2002). Dorsal anterior cingulate cortex: A role in reward-based decision making. Proceedings of the National Academy of Sciences, 99, 523-528.

Calvert, G. A., Campbell, R., \& Brammer, M. J. (2000). Evidence from functional magnetic resonance imaging of crossmodal binding in the human heteromodal cortex. Current Biology, 10, 649-657.

Calvert, G. A., Hansen, P. C., Iversen, S. D., \& Brammer, M. J. (2001). Detection of audio-visual integration sites in humans by application of electrophysiological criteria to the BOLD effect. NeuroImage, 14, 427-438.

Carr, L., IACObOni, M., Dubeau, M. C., Mazziotta, J. C., \& Lenzi, G. L. (2003). Neural mechanisms of empathy in humans: A relay from neural systems for imitation to limbic areas. Proceedings of the National Academy of Sciences, 100, 5497-5502.

CRAIG, A. D. (2003). Interoception: The sense of the physiological condition of the body. Current Opinion in Neurobiology, 13, 500-505.

Davis, K. D., Hutchison, W. D., LozANO, A. M., \& DostrovsKy, J. O. (1994). Altered pain and temperature perception following cingulotomy and capsulotomy in a patient with schizoaffective disorder. Pain, 59, 189-199.

Davis, K. D., TaYlor, S. J., Crawley, A. P., Wood, M. L., \& Mikulis, D. J. (1997). Functional MRI of pain- and attention-related activations in the human cingulate cortex. Journal of Neurophysiology, $\mathbf{7 7}$, 3370-3380.

DeCETy, J., \& Chaminade, T. (2003). Neural correlates of feeling sympathy. Neuropsychologia, 41, 127-138.

DeVInsKY, O., Morrell, M. J., \& VoGT, B. A. (1995). Contributions of anterior cingulate cortex to behavior. Brain, 118, 279-306.

di Pellegrino, G., Fadiga, L., Fogassi, L., Gallese, V., \& RizzoLATTI, G. (1992). Understanding motor events: A neurophysiological study. Experimental Brain Research, 91, 176-180.

Downar, J., Crawley, A. P., Mikulis, D. J., \& Davis, K. D. (2002). A cortical network sensitive to stimulus salience in a neutral behavioral context across multiple sensory modalities. Journal of Neurophysiology, 87, 615-620.

Eisenberger, N. I., Lieberman, M. D., \& Williams, K. D. (2003). 
Does rejection hurt? An fMRI study of social exclusion. Science, 302, 290-292.

Forman, S. D., Cohen, J. D., Fitzgerald, M., Eddy, W. F., Mintun, M. A., \& NoLL, D. C. (1995). Improved assessment of significant activation in functional magnetic resonance imaging (fMRI): Use of a cluster-size threshold. Magnetic Resonance in Medicine, 33, 636-647.

Francis, S. T., Kelly, E. F., Bowtell, R., Dunseath, W. J., Folger, S. E., \& McGlone, F. (2000). fMRI of the responses to vibratory stimulation of digit tips. NeuroImage, 11, 188-202.

Friston, K. J., Worsley, K. J., Frackowiak, R. S. J., Mazziotta, J., \& Evans, A. C. (1994). Assessing the significance of focal activations using their spatial extent. Human Brain Mapping, 1, 214-220.

GALLESE, V. (2001). The shared manifold hypothesis: From mirror neurons to empathy. Journal of Consciousness Studies, 8, 33-50.

Gallese, V. (2003). The manifold nature of interpersonal relations: The quest for a common mechanism. Philosophical Transactions of the Royal Society of London: Series B, 358, 517-528.

Gelnar, P. A., Krauss, B. R., SzeverenYi, N. M., \& ApKarian, A. V. (1998). Fingertip representation in the human somatosensory cortex: An fMRI study. NeuroImage, 7, 261-283.

Hadland, K. A., Rushworth, M. F. S., Gaffan, D., \& Passingham, R. E. (2003a). The anterior cingulate and reward-guided selection of actions. Journal of Neurophysiology, 89, 1161-1164.

Hadland, K. A., Rushworth, M. F. S., Gaffan, D., \& Passingham, R. E. (2003b). The effect of cingulate lesions on social behavior and emotion. Neuropsychologia, 41, 919-931.

Hari, R., Forss, N., Avikainen, S., KirvesKari, E., Salenius, S., \& Rizzolatti, G. (1998). Activation of human primary motor cortex during action observation: A neuromagnetic study. Proceedings of the National Academy of Sciences, 95, 15061-15065.

HikosaKa, K. (1997). Responsiveness of neurons in the posterior inferotemporal cortex to visual patterns in the macaque monkey. Behavioural Brain Research, 89, 275-283.

Hofbauer, R. K., Rainville, P., Duncan, G. H., \& Bushnell, M. C. (2001). Cortical representation of the sensory dimension of pain. Journal of Neurophysiology, 86, 402-411.

HSIEH, J.-C., STONe-Elander, S., \& InGVAR, M. (1999). Anticipatory coping of pain expressed in the human anterior cingulate cortex: A positron emission tomography study. Neuroscience Letters, 262, 61-64.

Hutchison, W. D., Davis, K. D., Lozano, A. M., Tasker, R. R., \& Dostrovsky, J. O. (1999). Pain-related neurons in the human cingulate cortex. Nature Neuroscience, 2, 403-405.

Iacoboni, M., Woods, R. P., Brass, M., BeKKering, H., Mazziotta, J. C., \& Rizzolatti, G. (1999). Cortical mechanisms of human imitation. Science, 286, 2526-2528.

JACKson, P. L., MeLtzoff, A. N., \& Decety, J. (2004, April). Perceiving others in painful situations activates the affective pain neural network. Paper presented at the annual meeting of the Cognitive Neuroscience Society, San Francisco.

Jenkinson, M., Bannister, P., Brady, M., \& Smith, S. (2002). Improved optimization for the robust and accurate linear registration and motion correction of brain images. NeuroImage, 17, 825-841.

KaAs, J. H., \& Collins, C. E. (2001). The organization of sensory cortex. Current Opinion in Neurobiology, 11, 498-504.

Keightley, M. L., Winocur, G., GrahaM, S. J., Mayberg, H. S. Hevenor, S. J., \& Grady, C. L. (2003). An fMRI study investigating cognitive modulation of brain regions associated with emotional processing of visual stimuli. Neuropsychologia, 41, 585-596.

Kenshalo, D. R., Iwata, K., Sholas, M., \& Thomas, D. A. (2000). Response properties and organization of nociceptive neurons in area 1 monkey primary somatosensory cortex. Journal of Neurophysiology, 84, 719-729.

KoYAMa, T., Kato, K., Tanaka, Y. Z., \& Mikami, A. (2001). Anterior cingulate activity during pain-avoidance and reward tasks in monkeys. Neuroscience Research, 39, 421-430.

Koyama, T., TanaKa, Y. Z., \& MiKami, A. (1998). Nociceptive neurons in the macaque anterior cingulate activate during anticipation of pain. NeuroReport, 9, 2663-2667.

Matelli, M., Luppino, G., \& Rizzolatti, G. (1991). Architecture of superior and mesial area 6 and the adjacent cingulate cortex in the macaque monkey. Journal of Comparative Neurology, 311, 445-462.

McGlone, F., Kelly, E. F., Trulsson, M., Francis, S. T., Westling, G.,
\& BowTELL, R. (2002). Functional neuroimaging studies of human somatosensory cortex. Behavioural Brain Research, 135, 147-158.

Melzack, R. (1999). From the gate to the neuromatrix. Pain, 6(Suppl.), S121-S126.

Milham, M. P., Banich, M. T., Claus, E. D., \& Cohen, N. J. (2003). Practice-related effects demonstrate complementary roles of anterior cingulate and prefrontal cortices in attentional control. NeuroImage, 18, 483-493.

Paus, T., Petrides, M., Evans, A. C., \& Meyer, E. (1993). Role of the human anterior cingulate cortex in the control of oculomotor, manual, and speech responses: A positron emission tomography study. Journal of Neurophysiology, 70, 453-469.

Peyron, R., Garcia-Larrea, L., Gregoire, M. C., Convers, P., Richard, A., Lavenne, F., Barral, F. G., Mauguiere, F., Michel, D., \& LAURENT, B. (2000). Parietal and cingulate processes in central pain. A combined positron emission tomography (PET) and functional magnetic resonance imaging (fMRI) study of an unusual case. Pain, 84, 7787

Peyron, R., Laurent, B., \& Garcia-Larrea, L. (2000). Functional imaging of brain responses to pain: A review and meta-analysis (2000). Clinical Neurophysiology, 30, 263-288.

Phillips, M. L., Young, A. W., Senior, C., Brammer, M., Andrews, C., Calder, A. J., Bullmore, E. T., Perrett, D. I., Rowland, D., Williams, S. C. R., Gray, J. A., \& David, A. S. (1997). A specific neural substrate for perceiving facial expressions of disgust. Nature, 389, 495-498.

Ploner, M., Freund, H. J., \& Schnitzler, A. (1999). Pain affect without pain sensation in a patient with a postcentral lesion. Pain, 81, 211-214.

Porro, C. A., Cettolo, V., Francescato, M. P., \& Baraldi, P. (2003). Functional activity mapping of the mesial hemispheric wall during anticipation of pain. NeuroImage, 19, 1738-1747.

Powell, T. P. S., \& Mountcastle, V. B. (1959). The cytoarchitecture of the postcentral gyrus of the monkey Macaca mulatta. Bulletin of Johns Hopkins Hospital, 105, 108-131.

Preston, S. D., \& DE WaAL, F. B. M. (2002). Empathy: Its ultimate and proximate bases. Behavioral \& Brain Science, 25, 1-20.

RAINVILLE, P. (2002). Brain mechanisms of pain affect and pain modulation. Current Opinion in Neurobiology, 12, 195-204.

Rainville, P., Carrier, B., Hofbauer, R. K., Bushnell, M. C., \& Duncan, G. H. (1999). Dissociation of sensory and affective dimensions of pain using hypnotic modulation. Pain, 82, 159-171.

Rainville, P., Duncan, G. H., Price, D. D., CARRIER, B., \& Bushnell, M. C. (1997). Pain affect encoded in human anterior cingulate but not somatosensory cortex. Science, 277, 968-971.

Ringler, R., Greiner, M., Kohl Loeffel, L., Handwerker, H. O., Forster, C. (2003). BOLD effects in different areas of the cerebral cortex during painful mechanical stimulation. Pain, 105, 445-453.

Rizzolatti, G., Fadiga, L., Fogassi, L., \& Gallese, V. (1999). Resonance behaviors and mirror neurons. Archives Italiennes de Biologie, 137, 85-100.

Rizzolatti, G., Fadiga, L., Gallese, V., \& Fogassi, L. (1996). Premotor cortex and the recognition of motor actions. Cognitive Brain Research, 3, 131-141.

RizZOLATTI, G., \& LUPPIno, G. (2001). The cortical motor system. Neuron, 31, 889-901.

Rushworth, M. F. S., Hadland, K. A., \& Passingham, R. E. (2003). The effect of cingulate cortex lesions on task switching and working memory. Journal of Cognitive Neuroscience, 15, 338-353.

Schnitzler, A., \& Ploner, M. (2000). Neurophysiology and functional neuroanatomy of pain perception. Journal of Clinical Neurophysiology, 17, 592-603.

Schnitzler, A., Seitz, R. J., \& Freund, H.-J. (2000). The somatosensory system. In A. W. Toga \& J. C. Mazziotta (Eds.), Brain mapping: The systems (pp. 291-329). San Diego: Academic Press.

SEWARDS, T. V., \& SEWARDs, M. A. (2002). The medial pain system: Neural representations of the motivational aspect of pain. Brain Research Bulletin, 59, 163-180.

Singer, T., Seymour, B., O’Doherty, J., Kaube, H., Dolan, R. J., \& FRITH, C. D. (2004). Empathy for pain involves the affective but not sensory components of pain. Science, 303, 1157-1162.

Smith, S. M. (2002). Fast robust automated brain extraction. Human Brain Mapping, 17, 143-155. 
Timmermann, L., Ploner, M., Haucke, K., Schmitz, F., Baltissen, R., \& SCHNITZLER, A. (2001). Differential coding of pain intensity in the human primary and secondary somatosensory cortex. Journal of Neurophysiology, 86, 1499-1503.

Vogt, B. A., Nimchinsky, E. A., Vogt, L. J., \& Hof, P. R. (1995). Human cingulate cortex: Surface features, flat maps, and cytoarchitecture. Journal of Comparative Neurology, 359, 490-506.

Vogt, B. A., \& Pandya, D. N. (1987). Cingulate cortex of the rhesus monkey: II. Cortical afferents. Journal of Comparative Neurology, 262, 271-289.

VoGT, B. A., \& SiKes, R. W. (2000). The medial pain system, cingulate cortex, and parallel processing of nociceptive information. Progress in Brain Research, 122, 223-235.

Walton, M. E., BanNerman, D. M., Alterescu, K., \& Rushworth, M. F. (2003). Functional specialization within medial frontal cortex of the anterior cingulate for evaluating effort-related decisions. Journal of Neuroscience, 23, 6475-6479.

Wicker, B., Keysers, C., Plailly, J., Royet, J. P., Gallese, V., \& Riz-
ZOLATTI, G. (2003). Both of us disgusted in my insula: The common neural basis of seeing and feeling disgust. Neuron, 40, 655-664.

WisPé, L. (1991). The psychology of sympathy. New York: Plenum. Woolrich, M. W., Ripley, B. D., Brady, M., \& SMith, S. M. (2001). Temporal autocorrelation in univariate linear modeling of $\mathrm{fMRI}$ data. NeuroImage, 14, 1370-1386.

Worsley, K. J., Evans, A. C., Marrett, S., \& Neelin, P. (1992). A three-dimensional statistical analysis for CBF activation studies in human brain. Journal of Cerebral Blood Flow Metabolism, 12, 900918.

YAMASAKI, H., LABAR, K. S., \& McCARThy, G. (2002). Dissociable prefrontal brain systems for attention and emotion. Proceedings of the National Academy of Sciences, 99, 11447-11451.

(Manuscript received October 16, 2003; revision accepted for publication May 4, 2004.) 